\title{
Einstein submanifolds with parallel mean curvature
}

\author{
Christos-Raent Onti
}

\begin{abstract}
We provide a classification of Einstein submanifolds in space forms with flat normal bundle and parallel mean curvature. This extends a previous result due to Dajczer and Tojeiro [3] for isometric immersions of Riemannian manifolds with constant sectional curvature.
\end{abstract}

\section{Introduction}

The study of isometric immersions of Riemannian manifolds with constant sectional curvature into space forms is a basic topic in submanifold theory that goes back to Cartan [1,2]. Several interesting results towards the classification of these immersions have been obtained ever since (see [4, 5, 9]). In particular, Dajczer and Tojeiro 3] provided a classification of all such isometric immersions with flat normal bundle and parallel mean curvature vector field.

A natural generalization of the concept of Riemannian manifolds with constant sectional curvature is the notion of manifolds with constant Ricci curvature, namely Einstein manifolds. Fialkow [7] and Thomas [15] initiated the study of isometric immersions of Einstein manifolds into space forms. Indeed, after the early work of Fialkow and Thomas, Ryan [14] gave a local classification of Einstein hypersurfaces in any space form. In arbitrary codimension, Di Scala [6] proved that Einstein real Kähler submanifolds of a Euclidean space are totally geodesic provided that they are minimal. The same conclusion still holds for minimal Einstein submanifolds with flat normal bundle in the Euclidean space (see [11]).

In the present paper, we classify isometric immersions of Einstein manifolds into a complete and simply connected Riemannian manifold $\mathbb{Q}_{c}^{N}$ of constant sectional curvature $c$ of arbitrary codimension, with flat normal bundle and parallel mean curvature vector field. Our result, that extends the aforementioned result of Dajczer and Tojeiro [3], is stated as follows:

2010 Mathematics Subject Classification. Primary 53B25; Secondary 53C40, 53C42.

Key words and phrases. Einstein submanifolds, parallel mean curvature, flat normal bundle, principal normals. 
Theorem 1 Let $f: M^{n} \rightarrow \mathbb{Q}_{c}^{N}, n \geq 3$, be an isometric immersion of a connected Einstein manifold with Ricci curvature $\lambda$, flat normal bundle and parallel mean curvature vector field. Then one of the following holds:

(i) The immersion $f$ is totally umbilical.

(ii) $\lambda=0=c$ and

$$
f\left(M^{n}\right) \subset \mathbb{S}^{1}\left(r_{1}\right) \times \cdots \times \mathbb{S}^{1}\left(r_{k}\right) \times \mathbb{R}^{n-k} \subset \mathbb{R}^{n+k} .
$$

(iii) $\lambda=0<c$ and

$$
f\left(M^{n}\right) \subset \mathbb{S}^{1}\left(r_{1}\right) \times \cdots \times \mathbb{S}^{1}\left(r_{n}\right) \subset \mathbb{S}_{c}^{2 n-1} \subset \mathbb{R}^{2 n},
$$

where $r_{1}^{2}+\cdots+r_{n}^{2}=1 / c$.

(iv) $\lambda=0>c$ and

$$
f\left(M^{n}\right) \subset \mathbb{H}^{1}\left(r_{1}\right) \times \mathbb{S}^{1}\left(r_{2}\right) \times \cdots \times \mathbb{S}^{1}\left(r_{n}\right) \subset \mathbb{H}_{c}^{2 n-1} \subset \mathbb{L}^{2 n},
$$

where $-r_{1}^{2}+r_{2}^{2}+\cdots+r_{n}^{2}=1 / c$.

(v) $\lambda=c(n-k)>0$ and

$$
f\left(M^{n}\right) \subset \mathbb{S}^{m_{1}}\left(\rho_{1}\right) \times \cdots \times \mathbb{S}^{m_{k}}\left(\rho_{k}\right) \subset \mathbb{S}_{c}^{n+k-1} \subset \mathbb{R}^{n+k},
$$

where $\rho_{i}=\sqrt{\left(m_{i}-1\right) / \lambda}, m_{i} \geq 2$ for all $1 \leq i \leq k$.

(vi) $f=j \circ g$, where $g$ is as in (ii), (iii), (iv) or (v) and $j$ is a totally umbilical inclusion.

Acknowledgments. The author would like to thank Prof. Theodoros Vlachos for his valuable suggestions and the referee for his close reading of the first draft of this paper which led to various improvements.

\section{Preliminaries}

Let $f: M^{n} \rightarrow \mathbb{Q}_{c}^{N}$ be an isometric immersion of an $n$-dimensional Riemannian manifold with Levi-Civita connection $\nabla$. The second fundamental form $\alpha$ of $f$ is a symmetric section of the vector bundle $\operatorname{Hom}\left(T M \times T M, N_{f} M\right)$, where $N_{f} M$ is the normal bundle of $f$. We say that $f$ is totally umbilical if

$$
\alpha(X, Y)=\langle X, Y\rangle H
$$


where $H$ is the mean curvature vector field.

A straightforward computation of the Ricci tensor gives using the Gauss equation for $f$ that

$$
\operatorname{Ric}(X, Y)=c(n-1)\langle X, Y\rangle+n\langle\alpha(X, Y), H\rangle-\sum_{i=1}^{n}\left\langle\alpha\left(X, X_{i}\right), \alpha\left(Y, X_{i}\right)\right\rangle, X, Y \in T M
$$

where $X_{1}, \ldots, X_{n}$ is a local orthonormal frame of the tangent bundle $T M$.

The immersion $f$ has flat normal bundle if the curvature tensor of the normal connection $\nabla^{\perp}$ of $N_{f} M$ vanishes. In this case, it is a standard fact (see [12]) that at any point $x \in M^{n}$ there exists a set of unique pairwise distinct normal vectors $\eta_{i}(x) \in N_{f} M(x), 1 \leq i \leq s=s(x)$, called the principal normals of $f$ at $x$. Moreover, there is an associated orthogonal splitting of the tangent space as

$$
T_{x} M=E_{1}(x) \oplus \cdots \oplus E_{s}(x)
$$

where

$$
E_{i}(x)=\left\{X \in T_{x} M: \alpha(X, Y)=\langle X, Y\rangle \eta_{i}(x) \text { for all } Y \in T_{x} M\right\} .
$$

Hence, the second fundamental form of $f$ at $x$ is given by

$$
\alpha(X, Y)=\sum_{i=1}^{s}\left\langle X^{i}, Y^{i}\right\rangle \eta_{i}(x), X, Y \in T_{x} M
$$

where $X^{i}$ denotes the $E_{i}(x)$-component of $X$.

The function $x \in M^{n} \rightarrow s(x) \in\{1,2, \ldots, n\}$ is lower semi-continuous. Hence, if $G_{k}$ denotes the interior of the subset $\left\{x \in M^{n}: s(x)=k\right\}$, then $\cup_{k=1}^{n} G_{k}$ is open and dense in $M^{n}$. On each $G_{k}$ the maps $x \in M^{n} \mapsto \eta_{i}(x), 1 \leq i \leq k$, are smooth vector fields called the principal normal vector fields of $f$ and the distributions $x \in M^{n} \mapsto E_{i}(x), 1 \leq i \leq k$, are smooth. The Codazzi equation on $G_{k}$ is easily seen to yield

$$
\left\langle\nabla_{X} Y, Z\right\rangle\left(\eta_{i}-\eta_{j}\right)=\langle X, Y\rangle \nabla_{Z}^{\perp} \eta_{i}
$$

and

$$
\left\langle\nabla_{X} V, Z\right\rangle\left(\eta_{j}-\eta_{l}\right)=\left\langle\nabla_{V} X, Z\right\rangle\left(\eta_{j}-\eta_{i}\right)
$$

for all $X, Y \in E_{i}, Z \in E_{j}$, and $V \in E_{l}$, where $1 \leq i \neq j \neq l \neq i \leq k$.

\section{$3 \quad$ Extrinsic product of immersions}

In this section, we recall the notion of extrinsic product of immersions in any space form (cf. [16]) that will be used in the proof of our main result. 
A map $f: M^{n} \rightarrow \mathbb{R}^{N}$ from a product manifold $M^{n}=\Pi_{i=1}^{k} M_{i}$ is called the extrinsic product of immersions $f_{i}: M_{i} \rightarrow \mathbb{R}^{m_{i}}, 1 \leq i \leq k$, if there exist an orthogonal decomposition $\mathbb{R}^{N}=\prod_{i=0}^{k} \mathbb{R}^{m_{i}}$, with $\mathbb{R}^{m_{0}}$ possibly trivial, such that $f$ is given by

$$
f(x)=\left(v, f_{1}\left(x_{1}\right), \ldots, f_{k}\left(x_{k}\right)\right)
$$

for all $x=\left(x_{1}, \ldots, x_{k}\right) \in M^{n}$ and $v \in \mathbb{R}^{m_{0}}$.

A map $f: M^{n} \rightarrow \mathbb{S}^{N}(r) \subset \mathbb{R}^{N+1}$ from a product manifold $M^{n}=\prod_{i=1}^{k} M_{i}$ into the sphere

$$
\mathbb{S}^{N}(r)=\left\{x \in \mathbb{R}^{N+1}:\|x\|=r\right\},
$$

is called the extrinsic product of immersions $f_{i}: M_{i} \rightarrow \mathbb{S}^{m_{i}-1}\left(r_{i}\right) \subset \mathbb{R}^{m_{i}}, 1 \leq i \leq k$, if there exist an orthogonal decomposition $\mathbb{R}^{N+1}=\prod_{i=0}^{k} \mathbb{R}^{m_{i}}$, with $\mathbb{R}^{m_{0}}$ possibly trivial, such that $f$ is given by

$$
f(x)=\left(v, f_{1}\left(x_{1}\right), \ldots, f_{k}\left(x_{k}\right)\right)
$$

for all $x=\left(x_{1}, \ldots, x_{k}\right) \in M^{n}$ with $v \in \mathbb{R}^{m_{0}}$ and

$$
\|v\|^{2}+\sum_{i=1}^{k} r_{i}^{2}=r^{2}
$$

We now consider extrinsic products in the hyperbolic space

$$
\mathbb{H}^{N}(r)=\left\{x=\left(x_{0}, \ldots, x_{N}\right) \in \mathbb{L}^{N+1}:\langle x, x\rangle=-r^{2}, x_{0}>0\right\},
$$

where $\mathbb{L}^{N+1}$ denotes the Lorentz space of dimension $N+1$. In this case, there are three different types of extrinsic products called hyperbolic, elliptic and parabolic.

A map $f: M^{n} \rightarrow \mathbb{H}^{N}(r) \subset \mathbb{L}^{N+1}$ from a product manifold $M^{n}=\Pi_{i=1}^{k} M_{i}$ is called the extrinsic product of hyperbolic type of immersions $f_{1}, \ldots, f_{k}$ if there exist an orthogonal decomposition

$$
\mathbb{L}^{N+1}=\mathbb{L}^{m_{1}} \times \Pi_{i=2}^{k+1} \mathbb{R}^{m_{i}},
$$

with $\mathbb{R}^{m_{k+1}}$ possibly trivial, and immersions

$$
f_{1}: M_{1} \rightarrow \mathbb{H}^{m_{1}-1}\left(r_{1}\right) \subset \mathbb{L}^{m_{1}} \text { and } f_{i}: M_{i} \rightarrow \mathbb{S}^{m_{i}-1}\left(r_{i}\right) \subset \mathbb{R}^{m_{i}}, 2 \leq i \leq k,
$$

such that $f$ is given by

$$
f(x)=\left(f_{1}\left(x_{1}\right), \ldots, f_{k}\left(x_{k}\right), v\right)
$$

for all $x=\left(x_{1}, \ldots, x_{k}\right) \in M^{n}$ with $v \in \mathbb{R}^{m_{k+1}}$ and

$$
-r_{1}^{2}+\sum_{i=2}^{k} r_{i}^{2}+\|v\|^{2}=-r^{2} .
$$


A map $f: M^{n} \rightarrow \mathbb{H}^{N}(r) \subset \mathbb{L}^{N+1}$ from a product manifold $M^{n}=\prod_{i=1}^{k} M_{i}$ is called the extrinsic product of elliptic type of immersions $f_{1}, \ldots, f_{k}$ if there exist an orthogonal decomposition

$$
\mathbb{L}^{N+1}=\prod_{i=1}^{k} \mathbb{R}^{m_{i}} \times \mathbb{L}^{m_{k+1}},
$$

a vector $v \in \mathbb{L}^{m_{k+1}}$, and immersions $f_{i}: M_{i} \rightarrow \mathbb{S}^{m_{i}-1}\left(r_{i}\right) \subset \mathbb{R}^{m_{i}}, 1 \leq i \leq k$, such that $f$ is given by

$$
f(x)=\left(f_{1}\left(x_{1}\right), \ldots, f_{k}\left(x_{k}\right), v\right)
$$

for all $x=\left(x_{1}, \ldots, x_{k}\right) \in M^{n}$ with

$$
\sum_{i=1}^{k} r_{i}^{2}+\langle v, v\rangle=-r^{2} .
$$

Finally, a map $f: M^{n} \rightarrow \mathbb{H}^{N}(r) \subset \mathbb{L}^{N+1}$ from a product manifold $M^{n}=\prod_{i=1}^{k} M_{i}$ is called the extrinsic product of parabolic type of immersions $f_{1}, \ldots, f_{k}$ if there exist $s \in\{1, \ldots, k\}$, an orthogonal decomposition

$$
\mathbb{L}^{N+1}=\mathbb{L}^{l+1} \times \Pi_{i=s+1}^{k+1} \mathbb{R}^{m_{i}},
$$

with $\mathbb{R}^{m_{k+1}}$ possibly trivial, and immersions

$$
f_{i}: M_{i} \rightarrow \mathbb{R}^{m_{i}}, 1 \leq i \leq s, \text { and } f_{j}: M_{j} \rightarrow \mathbb{S}^{m_{j}-1}\left(r_{j}\right) \subset \mathbb{R}^{m_{j}}, s+1 \leq j \leq k \text { if } s<k,
$$

such that $f$ is given by

$$
f(x)=\left(\mathrm{i}\left(f_{1}\left(x_{1}\right), \ldots, f_{s}\left(x_{s}\right)\right), f_{s+1}\left(x_{s+1}\right), \ldots, f_{k}\left(x_{k}\right), v\right)
$$

for all $x=\left(x_{1}, \ldots, x_{k}\right) \in M^{n}$ with $v \in \mathbb{R}^{m_{k+1}}$. Here

$$
\mathrm{i}: \prod_{i=1}^{s} \mathbb{R}^{m_{i}}=\mathbb{R}^{l-1} \rightarrow \mathbb{H}^{l}\left(r_{1}\right) \subset \mathbb{L}^{l+1}
$$

denotes an umbilical inclusion with

$$
-r_{1}^{2}+\sum_{i=2}^{k} r_{i}^{2}+\|v\|^{2}=-r^{2} .
$$

Let $f: M^{n} \rightarrow \mathbb{Q}_{c}^{N}$ be an isometric immersion of a Riemannian manifold. If $M^{n}=$ $\prod_{i=1}^{k} M_{i}$ is a product manifold then the second fundamental form $\alpha$ is said to be adapted to the product structure of $M^{n}$ if

$$
\alpha\left(X_{i}, X_{j}\right)=0 \text { for all } X_{i} \in T M_{i}, X_{j} \in T M_{j} \text { with } 1 \leq i \neq j \leq k,
$$

where the tangent bundles $T M_{i}$ are identified with the corresponding tangent distributions to $M^{n}$. The next result, which is due to Moore [8] for the case $c=0$ and to Molzan [10,13] for the case $c \neq 0$, shows that products of isometric immersions are characterized by this property among isometric immersions of Riemannian products.

Theorem 2 Let $f: M^{n} \rightarrow \mathbb{Q}_{c}^{N}$ be an isometric immersion of a Riemannian product manifold $M^{n}=\prod_{i=1}^{k} M_{i}$ with adapted second fundamental form. Then $f$ is an extrinsic product of isometric immersions. 


\section{The proof}

Let $f: M^{n} \rightarrow \mathbb{Q}_{c}^{N}$ be an isometric immersion as in Theorem 1, In the following we are working on an open subset $G_{k}$ with $k \geq 2$.

Lemma 3 Around every point $p \in G_{k}$ there is a neighborhood $U$ that is a Riemannian product of Riemannian manifolds $M_{1}, \ldots, M_{k}$. Moreover, $\left.f\right|_{U}$ is the extrinsic product of totally umbilical isometric immersions $f_{1}, \ldots, f_{k}$.

Proof: We claim that each distribution $E_{i}, 1 \leq i \leq k$, is parallel, that is

$$
\nabla_{X} Y \in E_{i} \text { for all } X \in T G_{k}, Y \in E_{i} \text { and } 1 \leq i \leq k
$$

First we prove that $\nabla_{X} Y \in E_{i}$ for all $X, Y \in E_{i}$ and $1 \leq i \leq k$. Indeed, from (4) we have that

$$
\left\langle\nabla_{X} Y, Z\right\rangle\left(\eta_{i}-\eta_{j}\right)=\langle X, Y\rangle \nabla_{Z}^{\perp}\left(\eta_{i}-\frac{n}{2} H\right)
$$

for any $X, Y \in E_{i}$ and $Z \in E_{j}$ with $j \neq i$. Thus, we obtain

$$
2\left\langle\nabla_{X} Y, Z\right\rangle\left\langle\eta_{i}-\eta_{j}, \eta_{i}-\frac{n}{2} H\right\rangle=\langle X, Y\rangle Z\left(\left\|\eta_{i}-\frac{n}{2} H\right\|^{2}\right) .
$$

Using (10), (3) and the hypothesis that $M^{n}$ is an Einstein manifold we have

$$
\left\|\eta_{i}-\frac{n}{2} H\right\|^{2}=\left\|\frac{n}{2} H\right\|^{2}-\lambda+c(n-1), 1 \leq i \leq k .
$$

Using (17) we observe that

$$
\left\langle\eta_{i}-\eta_{j}, \eta_{i}-\frac{n}{2} H\right\rangle=\left\|\eta_{i}-\frac{n}{2} H\right\|^{2}-\left\langle\eta_{i}-\frac{n}{2} H, \eta_{j}-\frac{n}{2} H\right\rangle \neq 0 .
$$

Then (6) implies that $\nabla_{X} Y \in E_{i}$ for all $X, Y \in E_{i}$ and any $1 \leq i \leq k$.

Now, we show that $\nabla_{X} Y \in E_{i}$ for all $X \in E_{j}$ and $Y \in E_{i}$ with $j \neq i$. To this aim, we consider $Y \in E_{i}, X \in E_{j}, Z \in E_{l} \subset E_{i}^{\perp}$ and distinguish the following two cases.

If $l=j$, then by using the previous argument, we have

$$
\left\langle\nabla_{X} Y, Z\right\rangle=-\left\langle Y, \nabla_{X} Z\right\rangle=0
$$

If $l \neq j$, then from (5) we obtain

$$
\left\langle\nabla_{X} Y, Z\right\rangle\left(\eta_{l}-\eta_{i}\right)=\left\langle\nabla_{Y} X, Z\right\rangle\left(\eta_{l}-\eta_{j}\right) .
$$

We claim that $\eta_{l}-\eta_{i}$ and $\eta_{l}-\eta_{j}$ are pointwise linearly independent. Indeed, we assume to the contrary that

$$
\eta_{l}-\eta_{i}=\mu\left(\eta_{l}-\eta_{j}\right)
$$


for some $\mu \in \mathbb{R} \backslash\{0\}$. Then

$$
(1-\mu)\left(\eta_{l}-\frac{n}{2} H\right)=\eta_{i}-\frac{n}{2} H-\mu\left(\eta_{j}-\frac{n}{2} H\right)
$$

By taking the norms and using (77) we obtain that $\eta_{i}=\eta_{j}$, which is a contradiction. Thus

$$
\left\langle\nabla_{X} Y, Z\right\rangle=0 \text { for all } X \in E_{j}, Y \in E_{i}, Z \in E_{l},
$$

with $l \neq i \neq j$. Therefore, from (8) and (91), we obtain that $\nabla_{X} Y \in E_{i}$ for all $X \in E_{j}$ and $Y \in E_{i}$ with $j \neq i$. This completes the proof of our claim.

Now, de Rham's theorem implies that around every point $p \in G_{k}$ there is a neighborhood $U$ that is the Riemannian product of the integral manifolds $M_{1}, \ldots, M_{k}$ of the distributions $E_{1}, \ldots, E_{k}$ respectively, through a point $q \in U$. Moreover, since the second fundamental form of $f$ is adapted, Theorem 2 implies that $\left.f\right|_{U}$ is an extrinsic product of isometric immersions $f_{1}, \ldots, f_{k}$, which due to (2) have to be totally umbilical.

Lemma 4 Let $m_{i}=\operatorname{dim} M_{i}$. Then the following holds:

$$
\left(m_{i}-1\right)\left(c+\left\|\eta_{i}\right\|^{2}\right)=\lambda, 1 \leq i \leq k
$$

Moreover, if $m_{i} \geq 2$ then the sectional curvature of $M_{i}$ is

$$
K_{M_{i}}=\frac{\lambda}{m_{i}-1}
$$

Furthermore, if $m_{i} \geq 2$ for all $1 \leq i \leq k$, then $\lambda>0$.

Proof: It follows from Lemma 3 and the Gauss equation that the principal normals $\eta_{1}, \ldots, \eta_{k}$ of $f$ satisfy

$$
\left\langle\eta_{i}, \eta_{j}\right\rangle=-c, 1 \leq i \neq j \leq k .
$$

Equation (10) follows from our assumption, (11) and (12). If $m_{i} \geq 2$ then (10) and the Gauss equation imply (11).

Now, suppose that $m_{i} \geq 2$ for all $1 \leq i \leq k$ and assume to the contrary that $\lambda \leq 0$. Then (10) implies that

$$
\left\|\eta_{i}\right\|^{2} \leq-c \text { for all } 1 \leq i \leq k
$$

and thus $c<0$. Therefore, from (12), (13) and the Cauchy-Schwarz inequality we obtain that

$$
-c=\left\langle\eta_{i}, \eta_{j}\right\rangle \leq\left\|\eta_{i}\right\|\left\|\eta_{j}\right\| \leq-c \text { for all } i \neq j .
$$

Hence, $\eta_{j}=\mu_{i j} \eta_{i}$ for some $\mu_{i j}>0$ and $1 \leq i \neq j \leq k$. From (12) and (13) we have that $\mu_{i j} \geq 1$ for all $1 \leq i \neq j \leq k$. Since $\eta_{j}=\mu_{i j} \eta_{i}=\mu_{i j} \mu_{j i} \eta_{j}$, it follows that $\mu_{i j} \leq 1$. Therefore, $\mu_{i j}=1$ which is a contradiction. 
Lemma 5 Assume that there exists $1 \leq i \leq k$ such that $m_{i}=1$. Then $U$ is flat.

Proof: The proof follows from Lemmas 3 and 4 ,

Proof of Theorem 1]: Let $f: M^{n} \rightarrow \mathbb{Q}_{c}^{N}, n \geq 3$, be an isometric immersion of a connected Einstein manifold with Ricci curvature $\lambda$, flat normal bundle and parallel mean curvature vector field. If $c \neq 0$, we always view $\mathbb{Q}_{c}^{N}$ as an umbilical hypersurface of the Euclidean space $\mathbb{R}^{N+1}$ or the Lorentzian space $\mathbb{L}^{N+1}$ according to the sign of $c$. In the following we are working on an open subset $G_{k}$.

If $k=1$, then $f$ is a totally umbilical immersion. In the sequel we assume that $k \geq 2$ and let $p \in G_{k}$. Then, according to Lemma 3 we have that there exists a neighborhood $U$ of $p$ that is a Riemannian product of Riemannian manifolds $M_{1}, \ldots, M_{k}$ and $\left.f\right|_{U}$ is an extrinsic product of totally umbilical isometric immersions $f_{1}, \ldots, f_{k}$.

We distinguish two cases.

If there exists $1 \leq i \leq k$ such that $m_{i}=1$ then the result follows from Lemma 5 and Theorem 1 of 3 ].

We now assume that $m_{i} \geq 2$ for all $1 \leq i \leq k$. If $c=0$, then each $f_{i}$ is an umbilical isometric immersion into $\mathbb{R}^{m_{i}+1}$, where $\overline{\mathbb{R}^{N}}=\mathbb{R}^{m} \times \prod_{i=1}^{k} \mathbb{R}^{m_{i}+1}$. Therefore, bearing in mind (11) we obtain that

$$
f(U) \subset \mathbb{S}^{m_{1}}\left(\rho_{1}\right) \times \cdots \times \mathbb{S}^{m_{k}}\left(\rho_{k}\right) \subset \mathbb{R}^{N}
$$

If $c>0$, then each $f_{i}$ is an umbilical isometric immersion into $\mathbb{S}^{m_{i}+1}\left(r_{i}\right) \subset \mathbb{R}^{m_{i}+2}$, where $\mathbb{R}^{N+1}=\mathbb{R}^{m} \times \Pi_{i=1}^{k} \mathbb{R}^{m_{i}+2}$. Thus, by using (11) we obtain that

$$
f(U) \subset \mathbb{S}^{m_{1}}\left(\rho_{1}\right) \times \cdots \times \mathbb{S}^{m_{k}}\left(\rho_{k}\right) \subset \mathbb{S}_{c}^{N}
$$

Finally, if $c<0$ then $f$ is the extrinsic product of umbilical isometric immersions $f_{1}, \ldots, f_{k}$ of either hyperbolic, elliptic or parabolic type.

If $f_{1}, \ldots, f_{k}$ is of hyperbolic type, then $f_{1}$ is an umbilical isometric immersion into $\mathbb{H}^{m_{1}+1}\left(r_{1}\right) \subset \mathbb{L}^{m_{1}+2}$ and each $f_{i}$ is an umbilical isometric immersion into $\mathbb{S}^{m_{i}+1}\left(r_{i}\right) \subset$ $\mathbb{R}^{m_{i}+2}, 2 \leq i \leq k$, where $\mathbb{L}^{N+1}=\mathbb{L}^{m_{1}+2} \times \prod_{i=2}^{k} \mathbb{R}^{m_{i}+2} \times \mathbb{R}^{m_{k+1}}$. Using (11) we obtain that

$$
f(U) \subset \mathbb{S}^{m_{1}}\left(\rho_{1}\right) \times \cdots \times \mathbb{S}^{m_{k}}\left(\rho_{k}\right) \subset \mathbb{H}_{c}^{N}
$$

Clearly, $f(U)$ is contained in a totally umbilical submanifold of $\mathbb{H}_{c}^{N}$ of positive sectional curvature.

If $f_{1}, \ldots, f_{k}$ is of elliptic type then each $f_{i}$ is an umbilical isometric immersion into $\mathbb{S}^{m_{i}+1}\left(r_{i}\right) \subset \mathbb{R}^{m_{i}+2}$, where $\mathbb{L}^{N+1}=\prod_{i=1}^{k} \mathbb{R}^{m_{i}+2} \times \mathbb{L}^{m}$. Bearing in mind (11) we obtain that

$$
f(U) \subset \mathbb{S}^{m_{1}}\left(\rho_{1}\right) \times \cdots \times \mathbb{S}^{m_{k}}\left(\rho_{k}\right) \subset \mathbb{H}_{c}^{N} .
$$

Clearly, $f(U)$ is contained in a flat totally umbilical submanifold of $\mathbb{H}_{c}^{N}$. 
If $f_{1}, \ldots, f_{k}$ is of parabolic type then there exist $s \leq k$ such that each $f_{i}$ is an umbilical isometric immersion into $\mathbb{R}^{m_{i}+1}$ for $1 \leq i \leq s$ and each $f_{j}$ is an umbilical isometric immersion into $\mathbb{S}^{m_{j}+1}\left(r_{j}\right) \subset \mathbb{R}^{m_{j}+2}$ for $s+1 \leq j \leq k$ if $s<k$, where $\mathbb{L}^{N+1}=$ $\mathbb{L}^{l} \times \prod_{i=s+1}^{k} \mathbb{R}^{m_{i}+2} \times \mathbb{R}^{m_{k+1}}$. Thus, from (11) we obtain that

$$
f(U) \subset \mathrm{i}\left(\Pi_{i=1}^{s} \mathbb{S}^{m_{i}}\left(\rho_{i}\right)\right) \times \Pi_{i=s+1}^{k} \mathbb{S}^{m_{i}}\left(\rho_{i}\right) \subset \mathbb{H}_{c}^{N}
$$

Again in this case $f(U)$ is contained in a flat totally umbilical submanifold of $\mathbb{H}_{c}^{N}$.

Finally, since $M^{n}$ is connected and the above different type of submanifolds cannot be smoothly attached we have that $f\left(M^{n}\right)$ is an open subset of one of the above and this completes the proof.

\section{References}

[1] E. Cartan, Sur les variétés de courbure constante d'un espace euclidien ou non-euclidien, Bull. Soc. Math. France 47 (1919), 125-160 (French).

[2] _ Sur les variétés de courbure constante d'un espace euclidien ou non-euclidien, Bull. Soc. Math. France 48 (1920), 132-208 (French).

[3] M. Dajczer and R. Tojeiro, Submanifolds of constant sectional curvature with parallel or constant mean curvature, Tohoku Math. J. (2) 45 (1993), no. 1, 43-49.

[4] _ Isometric immersions and the generalized Laplace and elliptic sinh-Gordon equations, J. Reine Angew. Math. 467 (1995), 109-147.

[5] — On compositions of isometric immersions, J. Differential Geom. 36 (1992), no. 1, 1-18.

[6] A. J. Di Scala, Minimal immersions of Kähler manifolds into Euclidean spaces, Bull. Lond. Math. Soc. 35 (2003), no. 6, 825-827.

[7] A. Fialkow, Hypersurfaces of a space of constant curvature, Ann. of Math. 39 (1938), no. 4, 762-785.

[8] J. D. Moore, Isometric immersions of riemannian products, J. Differential Geom. 5 (1971), 159-168.

[9] _ Submanifolds of constant positive curvature I, Duke Math. J. 44 (1977), no. 2, 449-484.

[10] S. Molzan, Extrinsische Producte und symmetrische Untermannigfaltigkeiten in Standardraumen konstanter und konstanter holomorpher Krümmung (1983). Doctoral Thesis, Koln.

[11] S. Nölker, Isometric immersions with homothetical Gauss map, Geom. Dedicata 34 (1990), no. 3, $271-280$.

[12] H. Reckziegel, Krümmungsflächen von isometrischen Immersionen in Räume konstante Krümmung, Math. Ann. 223 (1976), 169-181.

[13] - Hypersurfaces with parallel Ricci tensor in spaces of constant curvature, Results Math. 27 (1995), no. 1-2, 113-116. Festschrift dedicated to Katsumi Nomizu on his 70th birthday (Leuven, 1994; Brussels, 1994).

[14] P. J. Ryan, Homogeneity and some curvature conditions for hypersurfaces, Tohoku Math. J. (2) 21 (1969), no. 3, 363-388. 
[15] T. Y. Thomas, On closed spaces of constant mean curvature, Amer. J. Math. 58 (1936), no. 4, 701-704.

[16] R. Tojeiro, A decomposition theorem for immersions of product manifolds, Proc. Edinb. Math. Soc. 59 (2016), 247-269.

Department of Mathematics, University of Ioannina, 45110 Ioannina, Greece E-mail address: chonti@cc.uoi.gr 